\title{
OPERATION OF A CHERENKOV COUNTER WITH AN ULTRAVIOLET PHOTOIONIZATION DETECTOR*
}

\author{
J. CHAPMAN, D. MEYER and R. THUN \\ University of Michigan, Physics Departmem. Ann Arbor. Michigan 48109. U.S.A.
}

Received 20 July 1978

We have built and successfully operated a Cherenkov counter employing the far ultraviolet region of the spectrum to photoionize benzene in a proportional chamber. The operating conditions for such a chamber are described. The results of tests in a beam of pions are shown.

\section{Introduction}

Cherenkov counters have played a major role in identifying particles in high energy physics. The usual configuration involves detecting the Cherenkov light from the visible region of the spectrum by using a photomultiplier. In some instances especially with large $4 \pi$ detectors surrounding the intersection of colliding beam accelerators the need to keep the photomultipliers out of the magnetic field of the detector and the desire for large solid angle coverage lead to very complex or impossible optical problems.

It has been suggested') that Cherenkov counters be built to detect the far ultraviolet component of the Cherenkov light by employing a proportional counter filled with a standard argon- $\mathrm{CO}_{2}$ gas mixture doped with a small amount of $\mathrm{C}_{6} \mathrm{H}_{6}$. The benzene is an excellent photon detector since it has a $50 \%$ photoionization efficiency in the wavelength interval from $1350 \AA$ to the transmission limit of LiF at $1050 \AA$. We describe here a counter which we have built and tested in a high energy particle beam using this technique.

\section{Intensity calculation}

The yield of photoelectrons per unit length of Cherenkov radiator is $\mathrm{d} \varphi / \mathrm{d} /=370 \int \varepsilon \sin ^{2} \theta \hbar \mathrm{d} \omega$, where $\hbar \omega$ is the photon energy in $\mathrm{eV}$ and $\theta$ is the Cherenkov angle. $/$ is in $\mathrm{cm} . \varepsilon$ is the efficiency for converting the photons to photoelectrons and also may include transmission and reflection losses. For photomultipliers with quartz tube face or wavelength shifters the highest values of $\mathrm{d} \varphi / \mathrm{d} l$ are $150 \sin ^{2} \theta$. In practical counters mirror reflectance and window transmission reduce this value by a small amount to perhaps $80-90 \%$ of the above value.

* Work supported by the U.S. Department of Energy.
The yield for a proportional counter using benzene for a photoionizing gas is slightly more complicated to estimate because the transmission losses of the counter window ${ }^{2}$ ), reflectance losses of mirrors $^{3}$ ), photoionization probability ${ }^{4}$ ) and in-
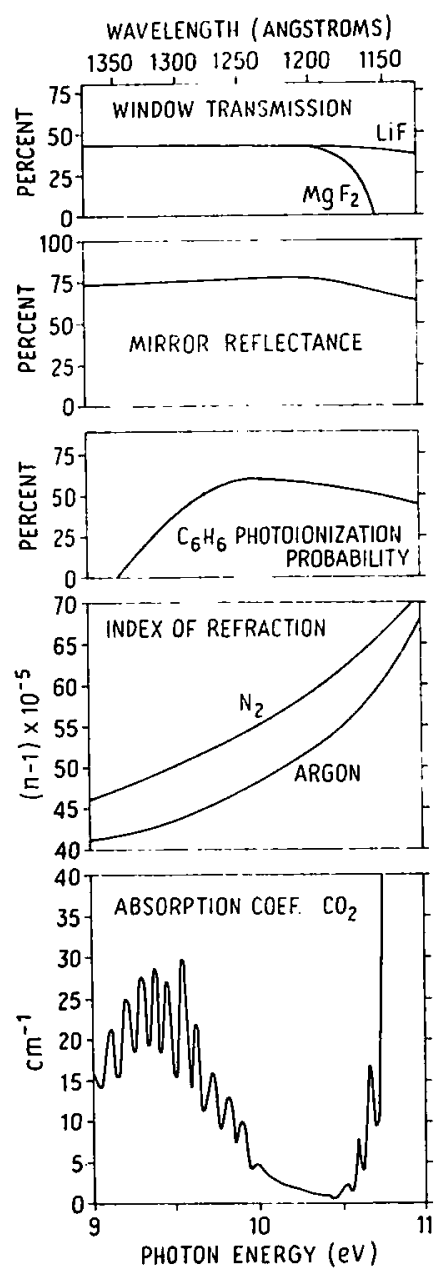

Fig. 1. Curves for window transmission, mirror reflectance, benzene photoionization, gas refractive index at $0^{\circ} \mathrm{C}, 1 \mathrm{~atm}$ and $\mathrm{CO}_{2}$ absorption as a function of photon energy. 


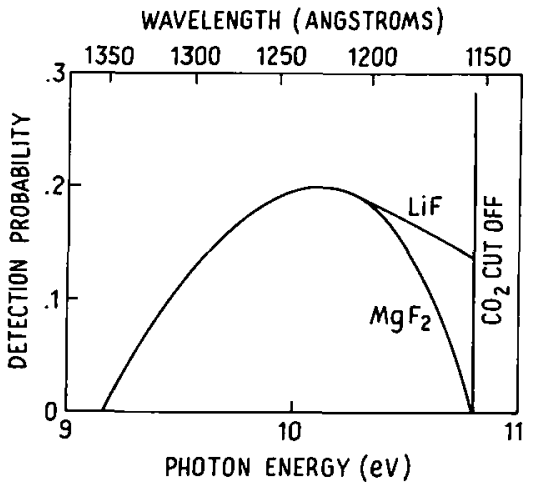

Fig. 2. A combination of the effects of window transmission, mirror reflectance and photoionization from fig. 1 .

dex of refraction ${ }^{5}$ ) (and hence $\theta$ ) of the Cherenkov radiator are all significantly dependent on photon energy. In addition the $\mathrm{CO}_{2}$ normally used as a quenching gas with argon in proportional chambers is absorbing below $1160 \AA^{6}$ ). In fig. 1 curves are shown for the measured values of these various components of the counter. The transmission curves are those for typical commercial samples, not the best attainable. They can be further degraded since both $\mathrm{MgF}_{2}$ and $\mathrm{LiF}$ are hygroscopic.

The $\mathrm{CO}_{2}$ absorption coefficient in such that for optimum photoionization conversion in an argon-10\% $\mathrm{CO}_{2}$-benzene gas mixture the benzene concentration should be high enough to photoionize in a few millimeters although at most wavelengths in the region of interest the $\mathrm{CO}_{2}$ absorption lengths are considerably longer. We will assume that the proportional chambers are so constructed that $\mathrm{CO}_{2}$ absorption is unimportant above $1160 \AA$ and complete $\mathrm{CO}_{2}$ absorption occurs below $1160 \AA$. In fig. 2 is shown the efficiency for photon detection from the combined effects of one mirror reflection, transmission through a $2 \mathrm{~mm}$ window and photoionization in benzene.

An integral over the curve of fig. 2 can now be made taking into account the fact that the index of refraction and hence the Cherenkov angle $\theta \cong\{2[(n-1)-(1-\beta)]\}^{!}$depends appreciably on photon energy. Neglecting the Cherenkov angle variation gives $\mathrm{d} \varphi / \mathrm{d} l \sim 100 \sin ^{2} \theta$. In a practical

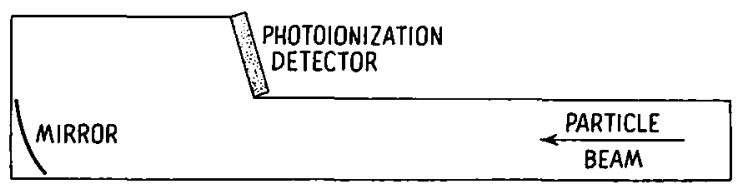

Fig. 3. Configuration of Cherenkov detector. counter this number is reduced by a number of factors that will be discussed later.

\section{Detector}

The configuration of the detector is shown in fig. 3. The spherical mirror which focuses the Cherenkov ring on the chamber was aluminized and coated $^{3}$ ) with $\mathrm{MgF}_{2}$. Two different mirrors were used. One had a focal length of $15 \mathrm{~cm}$ and a measured reflextivity of $78 \%$ at $1216 \AA$, the other a $30 \mathrm{~cm}$ focal length and a reflectivity of $65 \%$. The Cherenkov radiator was prepurified grade nitrogen or argon. An externally adjustable baffle made it possible to quickly change the length of the Cherenkov radiator viewed by the proportional chamber. Most measurements were made with the baffle out giving a $90 \mathrm{~cm}$ path for the long focal length mirror and $75 \mathrm{~cm}$ for the short focal length mirror. By coincidence the Cherenkov radiator lengths just compensate the reflectivity differences thus giving the same number of reflected photons in the two geometries.

Several different proportional chamber geometries were used (fig. 4). The window on all of the chambers was $4.5 \mathrm{~cm}$ in diameter. One chamber consisted of 4 square cells $1.27 \mathrm{~cm}$ on a side, the other of 7 cells $0.65 \mathrm{~cm}$ on a side. A grid of $0.08 \mathrm{~mm}$ diameter wire formed one side of the chamber. The window was in contact with this grid. Wire spacing of $0.8 \mathrm{~mm}, 1.6 \mathrm{~mm}$, and $3.2 \mathrm{~mm}$ were tried. The transmission of the $\mathrm{MgF}_{2}$ window was measured as $40 \%$ at $1216 \AA$. A LiF window with the same measured transmission was also used. The sense wires were $25 \mu \mathrm{m}$ gold plated tungsten.

The proportional counter gas was $90 \%$ argon, $10 \% \mathrm{CO}_{2}$. The photoionizing chemical was added by passing a portion of the argon- $\mathrm{CO}_{2}$ through benzene.

Because so few photoionization electrons were expected it was desirable to have a combined sig-

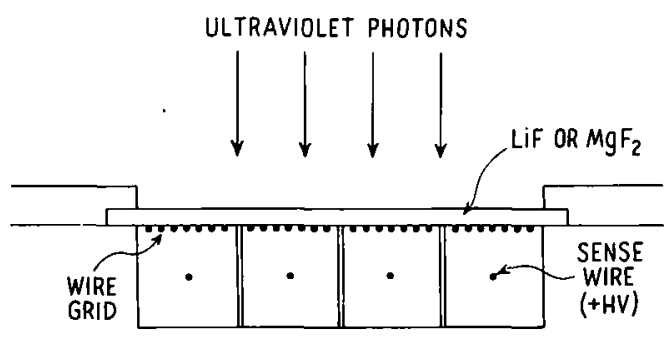

Fig. 4. Proportional chamber geometry. 
nal amplification of the proportional chamber plus external amplifier which was large enough to give high efficiency detection of single photoelectrons. A standard commercial preamplifier with a FET input stage was used. We estimate the noise level of this amplifier corresponded to a pulse of approximately $10^{4}$ electrons from the proportional chamber. A discriminator was set to pick up all pulses a factor of 2 larger than the noise pulses. The background rate under these conditions was a few counts per second.

\section{Results}

The entire counter was first tested in the laboratory. A small hole was drilled in the front of the counter and covered with a $\mathrm{MgF}_{2}$ window. A source of Lyman $\alpha$-radiation from a hydrogen discharge lamp provided $1216 \AA$ ultraviolet light which bounced off the mirror into the proportional chamber.

Even without benzene some electrons from ultraviolet photons were observed. These varied from $5 \%$ to $20 \%$ of those observed with the optimum benzene concentrations, the percentage depending on the previous history of the chamber. The photoelectric effect on the chamber walls is evidently substantial. By varying the chamber voltage or the amplifier gain it was possible to obtain a plateau showing that when no benzene was present, single photoelectron cascades were detected efficiently.

Benzene was then added to the proportional chamber and its concentration was varied to optimize photoionization. The addition of even small amounts of benzene changed the operating conditions of the chamber. To prevent very long pulses, presumably caused by ultraviolet photons from the cascade starting new avalanches by photoionizing

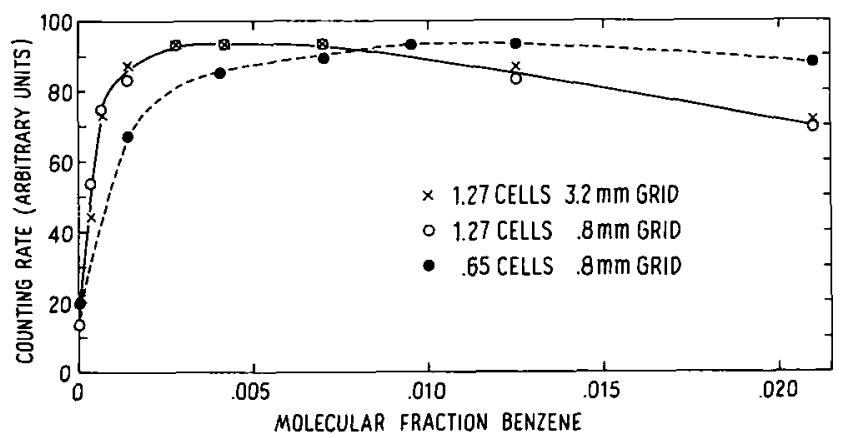

Fig. 5. Counting rate as a function of benzene concentration for three chamber geometries. the benzene, it was necessary to run the chamber at voltages $150 \mathrm{~V}$ lower than for the no benzene condition. Under these conditions no plateau could be reached and we estimate that at least $25 \%$ of the single photoelectrons were missed. Lower noise preamplifiers should solve this problem.

The results of these tests are shown in fig. 5 for the two chamber cell sizes and two grid spacings. As expected the $1.27 \mathrm{~cm}$ cells show saturation of counting rate at approximately half the benzene concentration of the $0.65 \mathrm{~cm}$ ones. The percentage of benzene needed to attain saturation agrees well with the distances calculated from the total cross section of benzene for ultraviolet photons $^{4}$ ). The grid spacing does not appreciably affect the shape of the curves. If a sufficient amount of benzene is added a fall in the counting rate is observed. The size and significance of this effect is however not easily interpreted because the operating conditions and hence the single photon detection efficiency of the chamber is afffected by the presence of benzene. Note that in a practical counter the benzene concentration is not critical but can be varied by a factor of 2 or more with little change in efficiency.

Following these laboratory tests the counter was tested in a pion beam at the ZGS at Argonne $\mathrm{Na-}$ tional Laboratory. In fig. 6 the threshold behavior of the counter is shown as a function of pion momentum. The curves are for a nitrogen Cherenkov radiator $90 \mathrm{~cm}$ long at $25^{\circ} \mathrm{C}$ for various pressures. If all single photoelectrons were detected with high efficiency the inefficiency of the counter would be that given by Poisson statistics for producing no

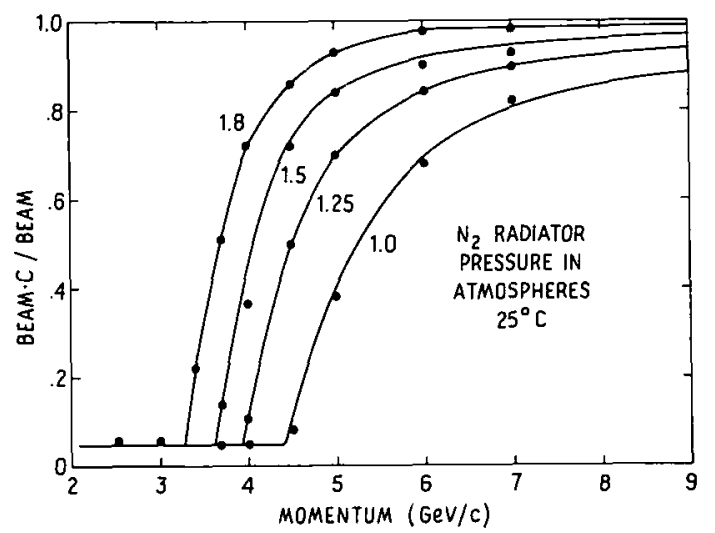

Fig. 6. Threshold behavior of $90 \mathrm{~cm}$ long counter in a beam of $\pi$ mesons. Dots are experimental points. Solid curves are predictions with $\varepsilon$ adjusted down by a factor of 2 from predicted values. 


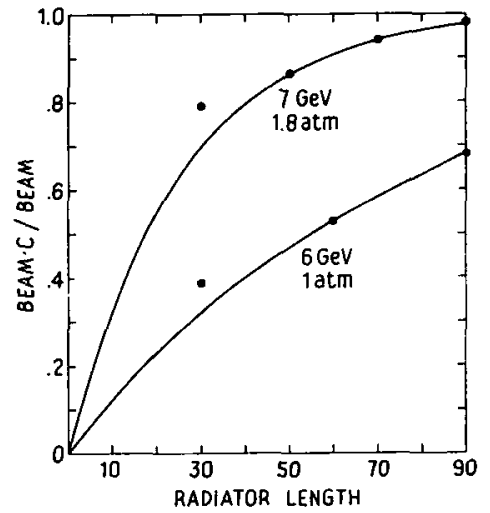

Fig. 7. Efficiency of counter as a function of Cherenkov radiator length. Dots are experimental points. Solid curves are predicted shapes.

photoelectrons, namely $\mathrm{e}^{-n}$, where $n$ is the average number of photoelectrons produced. Theoretical curves obtained by fitting the experimental points with $\varepsilon$ as a parameter are shown as solid lines. The test with a LiF window showed no substantial difference in counter efficiency from that for $\mathrm{MgF}_{2}$. The value of $\varepsilon$ thus obtained is a factor of 2 lower than that expected from an integration of fig. 2 over photon energy when the $90 \%$ open area of the window grid is considered. We thus observe $\mathrm{d} \varphi / \mathrm{d} l \approx 50 \sin ^{2} \theta$ photoelectrons per $\mathrm{cm}$.

To test the possibility that absorption in the Cherenkov gas radiator caused a significant loss of photons, a baffle was used to vary the effective length of the Cherenkov radiator. The results together with curves expected from photon statistics and no absorption are shown in fig. 7. There is some evidence for absorption but the results are not consistent with a simple explanation and more study is needed.

One problem that was anticipated was that scintillation light would be detected thus making the detector useless as a Cherenkov counter. When using very pure argon in the Cherenkov counter $17 \%$ of the pions were detected below threshold. With nitrogen or argon with a small nitrogen impurity this effect was much reduced so that approxiametely $5 \%$ of the beam particles were detected below threshold. It is known that the beam contains several percent electrons. Whether the residual detected particles are from delta rays, scin- tillation light or stray higher energy mesons has not yet been determined.

\section{Conclusions}

Our tests have demonstrated that using the ultraviolet part of the Cherenkov spectrum with photoionization techniques is a practical means of particle identification in situations where high magnetic fields or complex optical geometries make use of phototubes difficult or impossible. The major limitation of the technique is the limited range of indices of refraction available. Only nitrogen, hydrogen, and the noble gases are transparent in the wavelength range of interest.

Our measurements give a value for the number of detected photoelectrons a factor of 2 less than predicted. This discrepancy is probably due to a collection of small effects such as gas absorption, inefficient single photoelectron detection, etc. ${ }^{*}$ which can be at least partially corrected by improvements in technique.

Even with no improvement of techniques from those demonstrated here a useful counter can be built. We are now building prototypes of a counter to separate $\pi$ 's from electrons below $4 \mathrm{GeV}$ and $\pi$ 's from K's above $4 \mathrm{GeV}$ to cover greater than $70 \%$ of $4 \pi$ solid angle for a PEP detector.

We would like to thank S. Gray, D. Jankowski, and $\mathrm{R}$. Klem as well as the crew of the ZGS for their help with the beam and J.C. Person for discussions of ultraviolet techniques.

* Note added in proof: T. Ypsilantis has informed us that recent measurements on $\mathrm{N}_{2}$ absorption would predict a factor of 2 loss under our conditions.

\section{References}

I) J. Seguinot and T. Ypsilantis, Nucl. Instr, and Meth. 142 (1977) 377

2) D. A. Palterson and W. H. Vaughn, J. Opt. Soc, Am. 53 (1963) 852; Harshaw Chem. Co., private communication; $R$. J. Davis, J. Opt. Soc. Am. 56 (1966) 837: D. F. Heath and P. A. Sacher, Appl. Optics 5 (1966) 937.

3) Acton Res. Corp., Acton, MA., U.S.A.

4) J. C. Person, J. Chem. Phys. 43 (1965) 2553.

5) G. I. Chaschina, V. I. Gladuschak and E. Y. Schreider, Opt. Spect. 24 (1968) 542.

6) E. C. Y. Inn, K. Watanabe and M. Zelikoff, J. Chem. Phys. 21 (1953) 1648. 